\title{
A Uniform Description of Perioperative Brain MRI Findings in Infants with Severe Congenital Heart Disease: Results of a European Collaboration
}

\author{
(D) R. Stegeman, (D) M. Feldmann, (D) N.H.P. Claessens, (D) N.J.G. Jansen, (D).M.P.J. Breur, (D).S. de Vries, (D). Logeswaran, (D). Reich, \\ (D) W. Knirsch, (D) R. Kottke, (D) C. Hagmann, (D) B. Latal, (D). Simpson, (D). Pushparajah, (D) A.F. Bonthrone, (DC.J. Kelly, \\ (D) S. Arulkumaran, (DM.A. Rutherford, (D)S.J. Counsell, and (D) M.J.N.L. Benders, for \\ the European Association Brain in Congenital Heart Disease Consortium
}

슬

\begin{abstract}
BACKGROUND AND PURPOSE: A uniform description of brain MR imaging findings in infants with severe congenital heart disease to assess risk factors, predict outcome, and compare centers is lacking. Our objective was to uniformly describe the spectrum of perioperative brain MR imaging findings in infants with congenital heart disease.
\end{abstract}

MATERIALS AND METHODS: Prospective observational studies were performed at 3 European centers between 2009 and 2019. Brain MR imaging was performed preoperatively and/or postoperatively in infants with transposition of the great arteries, singleventricle physiology, or left ventricular outflow tract obstruction undergoing cardiac surgery within the first 6 weeks of life. Brain injury was assessed on T1, T2, DWI, SWI, and MRV. A subsample of images was assessed jointly to reach a consensus.

RESULTS: A total of 348 MR imaging scans (180 preoperatively, 168 postoperatively, 146 pre- and postoperatively) were obtained in 202 infants. Preoperative, new postoperative, and cumulative postoperative white matter injury was identified in $25 \%$, $30 \%$, and $36 \%$; arterial ischemic stroke, in $6 \%, 10 \%$, and $14 \%$; hypoxic-ischemic watershed injury in $2 \%$, $1 \%$, and $1 \%$; intraparenchymal cerebral hemorrhage, in $0 \%, 4 \%$, and $5 \%$; cerebellar hemorrhage, in $6 \%, 2 \%$, and $6 \%$; intraventricular hemorrhage, in $14 \%, 6 \%$, and $13 \%$; subdural hemorrhage, in $29 \%, 17 \%$, and $29 \%$; and cerebral sinovenous thrombosis, in $0 \%, 10 \%$, and $10 \%$, respectively.

CONCLUSIONS: A broad spectrum of perioperative brain MR imaging findings was found in infants with severe congenital heart disease. We propose an MR imaging protocol including T1-, T2-, diffusion-, and susceptibility-weighted imaging, and MRV to identify ischemic, hemorrhagic, and thrombotic lesions observed in this patient group.

ABBREVIATIONS: AIS = arterial ischemic stroke; CHD = severe congenital heart disease; CSVT = cerebral sinovenous thrombosis; IVH = intraventricular hemorrhage; $\mathrm{KCL}=$ St. Thomas' Hospital London; LVOTO = left ventricular outflow tract obstruction; SVP = single ventricle physiology; TGA $=$ transposition of the great arteries; UCZ = University Children's Hospital Zurich; WKZ = Wilhelmina Children's Hospital Utrecht; WMI = white matter injury

$\mathbf{T}$ he incidence of patients with severe congenital heart disease (CHD), presenting as severely ill and requiring expert cardiologic care in the neonatal period or early infancy is around $3 / 1000$

Received April 6, 2021; accepted after revision July 19.

From the Departments of Neonatology (R.S., N.H.P.C., L.S.d.V., M.J.N.L.B.), Pediatric Intensive Care (R.S., N.H.P.C., N.J.G.J.), and Pediatric Cardiology (R.S., N.H.P.C., J.M.P.J.B.), Wilhelmina Children's Hospital, UMC Utrecht, Utrecht, the Netherlands; Utrecht Brain Center (R.S., L.S.d.V., M.J.N.L.B.), UMC Utrecht, Utrecht University, Utrecht, the Netherlands; Child Development Center (M.F., B.L.), Division of Pediatric Cardiology (W.K.), Pediatric Heart Center, Department of Diagnostic Imaging (R.K.), and Department of Neonatology and Pediatric Intensive Care (C.H.), University Children's Hospital Zurich, Zurich, Switzerland; Department of Pediatrics (N.J.G.J.), Beatrix Children's Hospital, UMC Groningen, Groningen, the Netherlands; Pediatric Heart Center (T.L., B.R.), University Hospital Giessen, Justus-LiebigUniversity Giessen, Giessen, Germany; Department of Pediatric Cardiology (J.S., K.P.), Evelina Children's Hospital London, London, UK; and Centre for the Developing Brain (K.P., A.F.B., C.J.K., S.A., M.A.R., S.J.C.), School of Biomedical Engineering and Imaging Sciences, King's College London, London, UK.

R. Stegeman and M. Feldmann shared first authorship.

S.J. Counsell and M.J.N.L. Benders shared last authorship. live births. ${ }^{1}$ Mortality among infants has declined in recent decades, and $90 \%$ of children with CHD now survive into adulthood. ${ }^{2,3}$

This research was funded by a Consolidator Grant of the European Society of Pediatric Research. It was also funded by the Hartekind Foundation and Vrienden van het Wilhelmina Kinderziekenhuis Foundation; the Medical Research Council UK (MR/ L011530/1); the British Heart Foundation (FS/15/55/31649); the Action Medical Research (GN2630); the Wellcome Engineering and Physical Sciences Research Council Center for Medical Engineering at King's College London (WT 203148/Z/16/Z); and the MäxiFoundation, the Anna Müller Grocholski Foundation, and the EMDO Foundation Zurich.

Paper previously presented, in part, as abstracts at: Annual Scientific Sessions of the Cardiac Neurodevelopmental Outcome Collaborative, October 11-13, 2019; Toronto, Ontario, Canada; and Congress of the European Academy of Pediatric Societies, October 16-19, 2020; Virtual.

Please address correspondence to M.J.N.L. Benders, MD, PhD, Department of Neonatology, UMC Utrecht, KE 04.123.1, PO Box 85909, 3508 AB, Utrecht, the Netherlands; e-mail: M.Benders@umcutrecht.nl

- Indicates open access to non-subscribers at www.ajnr.org

Indicates article with online supplemental data.

http://dx.doi.org/10.3174/ajnr.A7328 
However, neurodevelopmental sequelae are a frequent long-term complication. $^{4}$

Consequently, in an endeavor to elucidate the underlying mechanisms of impaired neurodevelopment, a number of studies have reported performance of brain MR imaging before and after open heart surgery in infants with CHD. A combined pattern of abnormal brain development and acquired brain injury has been found. ${ }^{5-7}$ The most frequently reported lesions on perioperative MR imaging include white matter injury (WMI) and focal strokes. ${ }^{8-11}$ In addition, other findings such as hypoxic-ischemic watershed injury, intraparenchymal hemorrhage, and cerebral sinovenous thrombosis (CSVT) have also been reported. ${ }^{12-15}$

The prevalence of brain lesions varies considerably across studies. ${ }^{11,13,16-26}$ The large variability in the prevalence of brain lesions might reflect differences in inclusion criteria and practices among centers, but also a lack of a standardization in scoring and reporting of perioperative brain MR imaging findings in infants with $\mathrm{CHD}{ }^{26}$

A standardized description of perioperative brain MR imaging findings is important to accurately characterize the risks and patterns of brain lesions in infants with CHD. It facilitates the combination of data across centers to assess differences in medical care to determine lesion severity in relation to risk factors and subsequent neurodevelopmental outcome and enables neuroprotective approaches to be evaluated. The aim of this study was to describe the spectrum and prevalence of perioperative brain MR imaging findings in infants with $\mathrm{CHD}$ in a consistent manner, in terms of number, location, signal intensity, size, and volume across 3 European centers.

\section{MATERIALS AND METHODS Study Design and Population}

Three prospective observational cohort studies were combined. Infants with severe $\mathrm{CHD}$ who underwent corrective or palliative cardiac surgery during the first 6 weeks of life in the respective centers at Wilhelmina Children's Hospital Utrecht (WKZ, 2016-2019), University Children's Hospital Zurich (UCZ, 2009-2019), and St. Thomas' Hospital London (KCL, 20142019) were eligible for inclusion. We considered cardiac surgery to include both median and lateral thoracotomies, with or without use of cardiopulmonary bypass. Brain MR imaging was performed pre- and/or postoperatively per clinical (WKZ) or research study (UCZ, KCL) protocol. Severe CHD types included transposition of the great arteries, single-ventricle physiology, or left ventricular outflow tract obstruction (such as aortic arch coarctation, hypoplasia with/without coarctation, interruption, valve stenosis, or hypoplastic left-heart complex). Infants with known or suspected genetic or syndromic disorders and other types of CHD were excluded. Clinical characteristics of the infants were collected prospectively at each center and subsequently combined. The respective institutional ethics research committees approved the studies (WKZ, No. 16-093; UCZ, KEK StV-23/619/04; KCL, 07/H0707/105). Parental informed consent was obtained for the use of clinically obtained data for research purposes (WKZ) or before study enrollment (UCZ, KCL). All methods were performed in accordance with relevant guidelines and regulations. The de-identified data will be made available upon reasonable request.

\section{Brain MR Imaging Protocols}

MR images were acquired on a 3T scanner (Philips Healthcare, Best, the Netherlands) using a 32-channel head coil in WKZ, a neonate-specific 32-channel head coil at KCL, and a 3T Signa HDxt (GE Healthcare) scanner with an 8-channel head coil in UCZ. Infants were swaddled in a vacuum cushion and received noise-protecting earplugs, and vital functions were monitored. In $\mathrm{WKZ}$, infants were scanned in natural sleep or, if necessary, sedated with oral chloral hydrate $(50 \mathrm{mg} / \mathrm{kg})$ during MR imaging or received continuous sedation when mechanically ventilated. ${ }^{26}$ In UCZ, infants underwent MR imaging in natural sleep when clinically stable. In KCL, MR imaging was performed in natural sleep. MR imaging protocols included T1, T2, DWI and SWI, and MRV. ${ }^{11,24,27}$ In UCZ, SWI and MRV were acquired when there was suspicion of hemorrhage on conventional imaging or sinovenous thrombosis needed to be confirmed. Details of MR imaging protocols are available in the Online Supplemental Data.

\section{Describing Perioperative Brain MR Imaging Findings}

The system of describing perioperative brain MR imaging findings was determined jointly by the European Association Brain in Congenital Heart Disease Consortium and was based on the injury scoring sheet by Beca et al. ${ }^{19}$ This adapted template was used in joint European MR imaging reviewing sessions to find a consensus on terminology, definitions, and scoring of brain MR imaging findings in infants with CHD. This uniform European description was then applied to score MR images of each cohort according to the scoring sheet presented in the Online Supplemental Data. All MR imaging findings were described irrespective of the potential pathologic significance and consequences for neurodevelopmental outcome. Type, number, size, volume, location, and signal intensity of brain MR imaging findings were examined. Postoperative brain findings were classified as new if preoperative MR imaging showed no corresponding findings, findings were in a different location, and/or there was an increase in size or number compared with the preoperative findings. Cumulative postoperative brain MR imaging findings included all infants with $\mathrm{CHD}$ with a postoperative MR imaging irrespective of the availability of a preoperative MR imaging.

WMI was defined as single or multiple lesions in the white matter without restriction of maximum lesion size, with high signal intensity on $\mathrm{T} 1$ and usually corresponding low signal intensity on $\mathrm{T} 2 .^{24}$ Lesion sizes in each subject were measured on the T1 image in the plane showing the largest diameter, and largest lesion size was reported. Absolute WMI volume in cubic millimeters was segmented and calculated on 3D T1 images using ITK-SNAP (www. itksnap.org) (KCL) or 3D Slicer (http://www.slicer.org) (WKZ, UCZ). ${ }^{28,29}$ Total brain volumes were automatically calculated on T2 images using neonatal-specific segmentation pipelines and were used to report the relative WMI burden (WMI volume/total brain volume). ${ }^{30,31}$ Absolute WMI volume was not assessed in 7/ $45(16 \%)$ infants with preoperative WMI and in 8/60 (13\%) with postoperative WMI, due to motion corruption on $3 \mathrm{D}$ T1 images. The relative WMI burden could not be assessed in 15/45 (33\%) infants with preoperative WMI and in 15/60 (25\%) infants with postoperative WMI as a result of movement artifacts on either the 
3D T1-weighted images (WMI volume segmentation) or the T2weighted images (total brain volume segmentation).

Arterial ischemic stroke (AIS) was defined as a homogeneous area of altered signal intensity on T1- and T2-weighted images with a specific arterial distribution involving cortical gray matter and/or the basal ganglia/thalamus. ${ }^{24,26}$ AIS was classified on the basis of the involved arterial territory: anterior, middle, or posterior cerebral artery or perforator branch (involving the basal ganglia/thalamus). Middle cerebral artery strokes were subcategorized as main, anterior, middle, posterior, or cortical branch. ${ }^{32}$ Corticospinal tracts were involved when the corona radiata, and/or the posterior limb of the internal capsule, and/or the cerebral peduncle were affected. Hypoxic-ischemic watershed injury was defined as diffuse ischemia in intervascular borderzones among arterial territories. ${ }^{26}$ Restricted diffusion, indicating recently acquired ischemic lesions, was assessed by high signal intensity on DWI and/or low signal on ADC images.

Hemorrhages (intraparenchymal cerebral/cerebellar, intraventricular [IVH], and subdural) were assessed using SWI, if available. For the intraparenchymal supratentorial hemorrhages, we included lesions of any size when the lesion was hypointense on the SWI. Cerebellar hemorrhages are single or multiple hemorrhages located within the cerebellum. ${ }^{24}$ The size of cerebellar hemorrhages was quantified by measuring the largest diameter in millimeters on the SWI. IVH grade I was defined as bleeding restricted to the germinal matrix or choroid plexus; grade II, as extension of blood into the ventricles without enlargement; grade III, as ventricles enlarged by accumulated blood; and grade IV, periventricular hemorrhagic infarction, was defined when IVH was accompanied by periventricular hemorrhagic necrosis. ${ }^{26,33}$

CSVT was defined as MRV proved with T1 correlation (in WKZ/KCL) or high suspicion on T1/T2 (UCZ). ${ }^{15}$

Examples of brain findings on preoperative MR imaging sequences in infants with severe congenital heart disease are shown in the Online Supplemental Data.

\section{RESULTS \\ Study Participants}

A total of 202 infants with severe CHD (131 males, 65\%) with a median gestational age of 39.0 weeks (interquartile range, 38.340.0 weeks) and a median birth weight of $3200 \mathrm{~g}$ (interquartile range, 2940-3648 g) ( $z$ score interquartile range $-0.16,-0.77-$ 0.48 ) were enrolled at 3 European centers and met the inclusion criteria. Details of demographic and clinical characteristics are presented in the Online Supplemental Data.

\section{Preoperative Brain MR Imaging Findings}

Preoperative MR imaging was performed in 180 infants with $\mathrm{CHD}$ at a median age of 6 days (interquartile range, 3-8 days) and postmenstrual age of 39.7 weeks (interquartile range, 38.940.9 weeks). WMI was found in 45 infants (25\%); AIS, in 11 (6\%); hypoxic-ischemic watershed injury, in 3 (2\%); cerebellar hemorrhage, in 10 (6\%); and IVH, in 25 (14\%). We also observed subdural hemorrhage in 53 patients (29\%). Details of preoperative brain $M R$ imaging findings are described in the Online Supplemental Data. Preoperatively, no lesions, 1 type of lesion, or $\geq 2$ types of lesions were observed in 110 (61\%), 50 (28\%), and 20 (11\%) infants with CHD, respectively (Figure).

\section{Postoperative Brain MR Imaging Findings (New Lesions)}

New postoperative brain MR imaging findings were assessed in 146 infants with $\mathrm{CHD}$ and serial pre- and postoperative MR images. New WMI was found in 43 infants (30\%); AIS, in 15 (10\%); cerebellar hemorrhage, in 3 (2\%); IVH, in 8 (6\%); and subdural hemorrhage, in 25 (17\%). Intraparenchymal cerebral hemorrhage $(n=6$, $4 \%)$ and CSVT $(n=15,10 \%)$ were exclusively observed postoperatively (Online Supplemental Data). Postoperatively, no new lesions, 1 type of new lesion, or $\geq 2$ types of new lesion were shown in 83 (57\%), 42 (29\%), and 21 (14\%) infants, respectively (Figure).

\section{Postoperative Brain MR Imaging Findings (Also Including Infants without Preoperative MR Imaging)}

Cumulative postoperative brain MR imaging findings were assessed in 168 infants with $\mathrm{CHD}$ at a median age of 22 days (interquartile range, 15-29 days), median postmenstrual age of 42.7 weeks (interquartile range, 41.2-43.8 weeks), and a median of 10 days (7-15) after surgery. WMI was observed in 60 infants (36\%); AIS, in 24 (14\%); hypoxic-ischemic watershed injury, in 2 (1\%); intraparenchymal cerebral hemorrhage, in $8(5 \%)$; cerebellar hemorrhage, in 10 (6\%); IVH, in 22 (13\%); subdural hemorrhage, in 48 (29\%); and CSVT, in 17 (10\%). Details of postoperative brain MR imaging findings are described in the Online Supplemental Data. Postoperatively, no cumulative lesions, 1 type of cumulative lesion, or $\geq 2$ types of cumulative lesion were present in 75 (45\%), 60 (36\%), and 33 (20\%) infants, respectively (Figure).

\section{New Postoperative Brain Lesions in Infants with and without Preoperative Brain Lesions}

Forty-eight percent of infants with preoperative brain lesions showed new lesions on postoperative MR imaging. Thirty-nine percent of infants without preoperative brain lesions had new lesions on postoperative MR imaging. In 51 infants (35\%), no brain lesions were observed on either preoperative and postoperative MR imaging (Online Supplemental Data).

\section{DISCUSSION}

The aims of this European collaborative study were to standardize the description and consistently report perioperative brain MR imaging findings in infants with CHD. We report results from the largest combined cohort of infants with severe CHD thus far and found a broad spectrum of ischemic, hemorrhagic, and thrombotic brain lesions. WMI was the most prevalent lesion type, and WMI and AIS were common on both pre- and postoperative MR imaging. Cerebellar, intraventricular, and subdural hemorrhages were mainly observed preoperatively. Intraparenchymal cerebral hemorrhages and CSVT were exclusively detected on postoperative MR imaging.

WMI was the predominant finding on both pre- and postoperative MR imaging as reported previously. ${ }^{8,9,11,13,19,24}$ The distribution of WMI that we observed matched the pattern previously reported. Kelly et $\mathrm{al}^{24}$ and Guo et $\mathrm{al}^{34}$ found WMI to be widespread throughout the whole brain, including some cases with involvement of the corona radiata. Total WMI volume on pre- 

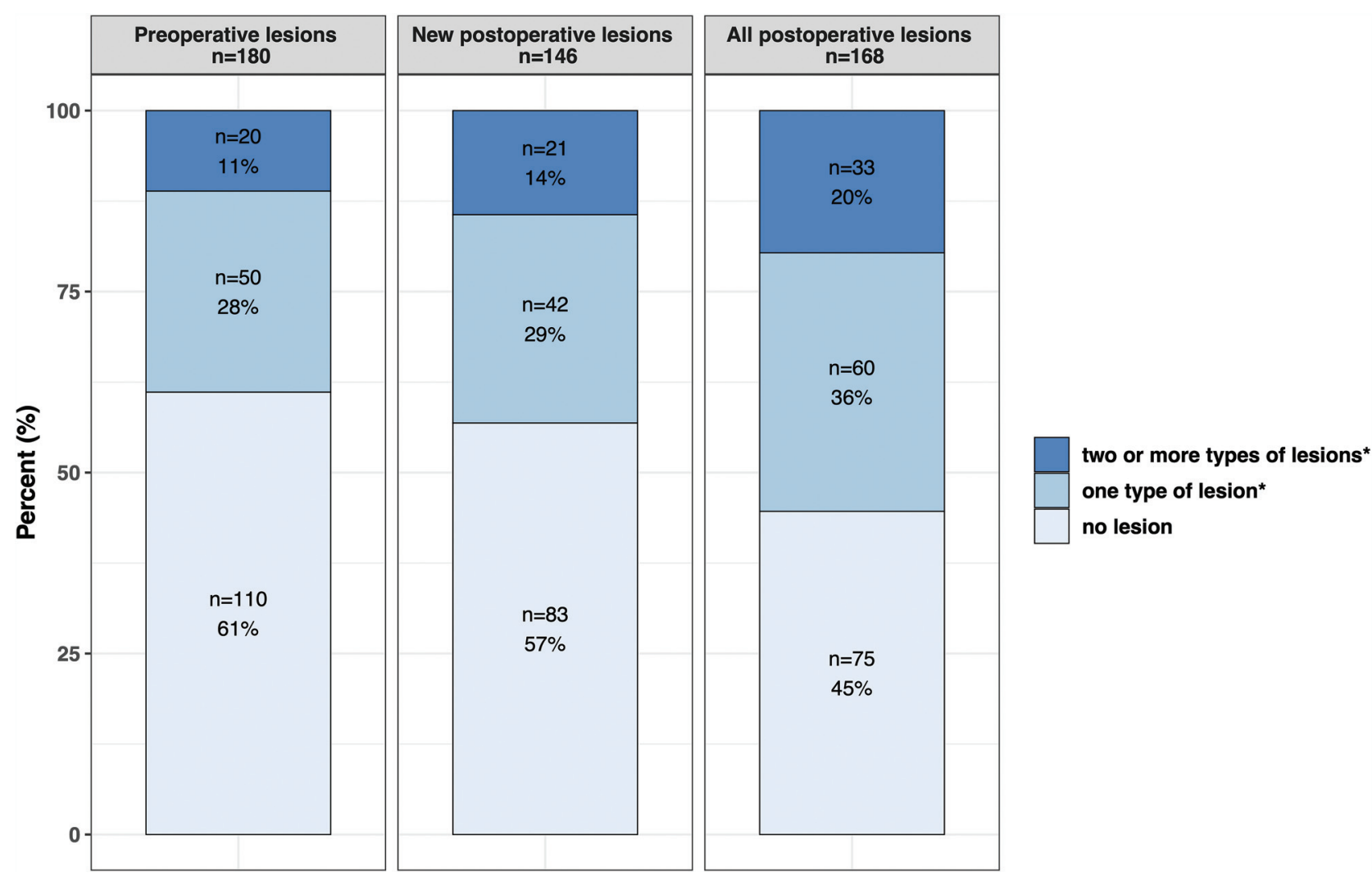

FIGURE. Proportion of infants with CHD with any kind of brain lesions. Any lesion included white matter injury, arterial ischemic stroke, hypoxic-ischemic watershed injury, intraparenchymal cerebral hemorrhage, cerebellar hemorrhage, intraventricular hemorrhage, and cerebral sinovenous thrombosis. Subdural hemorrhage was recorded but was not considered brain injury, being extra-axial and given its frequent occurrence in the healthy neonatal population.

and postoperative MR imaging in our European cohorts was in a similar range as reported recently in a multicenter analysis, suggesting that our findings might reflect the general pattern of WMI in neonates with CHD. ${ }^{34}$ The mechanisms underpinning WMI are not entirely clear, but it is possible that focal ischemic injury such as a single, large white matter lesion may have a thromboembolic origin, while multifocal WMI is probably caused by acute or chronic hypoxia-ischemia in infants with CHD. In a recent study by Claessens et al, ${ }^{26}$ focal injury (stroke, single white matter lesion) was more frequently seen after balloon atrial septostomy and associated with intraoperative, selective cerebral perfusion, while multifocal injury (watershed, WMI) was associated with low cardiac output syndrome. In our cohort, we recognize that thromboembolic strokes possibly caused single, large ischemic lesions, often confirmed on DWI or T1 as high signal, that exclusively affected the white matter.

AIS was identified more frequently on postoperative MR imaging compared with preoperative MR imaging, while hypoxic-ischemic watershed injury was rare, as reported previously. ${ }^{10,12,13} \mathrm{~A}$ wide spectrum of AIS was observed with different arterial distributions, sizes, and ages of lesions as indicated by diffusion restriction, which was in line with previous results by Chen et al. ${ }^{10}$ Most interesting, the middle cerebral artery branches were most frequently affected preoperatively, while the specific subtype of focal perforator strokes in the basal ganglia/thalamus region, including the posterior and middle cerebral artery branches, were most common on postoperative MR imaging. Preoperative AIS may be associated with balloon atrial septostomy, while selective cerebral perfusion has previously been associated with deep gray matter infarctions postoperatively. ${ }^{17,26,35}$ AIS with diffusion restriction and without clear signal intensity alterations on T1- and T2-weighted images, indicative of recent injury, was more often observed on pre- compared with postoperative MR imaging, presumably because preoperative AIS was still visible on postoperative conventional T1- and T2-weighted images, while DWI and ADC had pseudonormalized. These findings show that infants with CHD are vulnerable to AIS at different time points from birth to the postoperative period. Thromboembolic events or cerebral hypoperfusion by low cardiac output might contribute to the observed patterns and timing of AIS. $^{10,26}$

Cerebellar hemorrhages, low-grade IVH, and subdural hemorrhages were mainly present on preoperative MR imaging, while intraparenchymal cerebral hemorrhages and CSVT were exclusively observed on postoperative MR imaging, findings possibly indicating differences in the underlying etiology. ${ }^{13-15,24,36-38}$ Subdural hemorrhage has been observed at a similar rate in asymptomatic term-born infants that underwent instrumental vaginal delivery and in other $\mathrm{CHD}$ populations and may be explained by a tendency toward more frequent use of instrumental vaginal delivery in labor complicated by $\mathrm{CHD}$ as suggested by CJ Kelly et al. ${ }^{2437-40}$ Perioperative disturbances in cerebral autoregulation and coagulation could be responsible for postoperative 
hemorrhages and thromboses such as CSVT. ${ }^{41}$ Previous studies have highlighted the importance of SWI to assess intraparenchymal hemosiderin foci because signal abnormalities are not always identified on conventional T1- or T2-weighted images. ${ }^{13,14}$ CSVT was found less often postoperatively compared with the study by Claessens et $\mathrm{al},{ }^{15}$ which might be explained by differences in the proportions of CHD types and differences or interim changes in anticoagulatory and perioperative approaches. ${ }^{41}$ In both studies, the transverse sinus was most affected. ${ }^{15}$

Variability in brain lesions between our and other cohorts may be due to differences in the proportions of CHD types studied, clinical approaches such as age at surgery, and MR imaging protocols including section thickness, in-plane resolution, and timing of imaging. A larger interval between birth and the operation is associated with an increased incidence of preoperative WMI in infants with transposition of the great arteries, while hypoxic-ischemic brain injury is more often present on postoperative MR imaging after neonatal compared with postneonatal or infant heart surgery. ${ }^{8,18,42}$ Longer times between the operation and postoperative MR imaging could impair the sensitivity of DWI and ADC to detect transient ischemic lesions. ${ }^{12,13}$

This study has some limitations: Infants in UCZ and KCL were scanned as part of a research study and only after parental consent was given, with the risk of selection bias in types of $\mathrm{CHD}$, while infants at WKZ were scanned as part of standard clinical care. Differences in the timing of pre- and postoperative MR imaging, the operation, and image resolution may affect the sensitivity to detect small brain lesions. SWI and MRV were not performed routinely at UCZ, which might have impaired the sensitivity to detect small parenchymal hemorrhages and CSVT and led to an underestimation of respective prevalence. It was not possible to determine which portion of newly detected postoperative brain lesions actually occurred between the day of preoperative MR imaging and the day of the operation. Quantification methods of WMI volume and total brain volume differed among sites, though these were only used to determine the burden of WMI in relation to the total brain volume.

\section{CONCLUSIONS}

A broad spectrum of pre- and postoperative brain MR imaging findings was found in infants with severe CHD. An MR imaging protocol including T1-, T2-, DWI/ADC, SWI, and MRV is required to identify ischemic, hemorrhagic, and thrombotic lesions. Applying this standardized consensus description of perioperative brain MR imaging findings will enable future studies to determine lesion type, location, and extent in relation to outcome, identify risk factors across and among centers, and evaluate neuroprotective strategies in individuals with severe CHD.

\section{ACKNOWLEDGMENTS}

We are indebted to the families who supported this study. The authors would like to thank Julia Gunn and her colleagues from the Royal Children's Hospital Melbourne for kindly providing their MR imaging scoring sheet as a template for our uniform description of perioperative brain MR imaging findings. WKZ:
We thank the Congenital Heart Disease LifeSpan Research group, which includes staff from the Departments of Obstetrics, Pediatric Heart Center, Pediatric Intensive Care, Neonatology, and Radiology. KCL: We thank the staff from the St. Thomas' Neonatal Intensive Care Unit; the Evelina Children's London Hospital Fetal and Paediatric Cardiology Departments; the Evelina London Paediatric Intensive Care Unit; the Centre for the Developing Brain at King's College London; our research radiographers; and our neonatal scanning team. UCZ: We thank Dr Ruth O'Gorman Tuura for the setup and technical supervision of the MR imaging protocol and Drs Oliver Kretschmar and Dave Hitendu for comments on the manuscript. We thank the staff from the Center for Magnetic Resonance Research; the Pediatric Heart Center; the Department of Intensive Care Medicine and Neonatology; and the Department of Diagnostic Imaging at the University Children's Hospital Zurich.

Disclosures: Raymond Stegeman-RELATED: Grant: European Society of Pediatric Research Grant, Grant: ZonMw CRUCIAL-TRIAL, Stichting Hartekind*, Comments: Linda de Vries-RELATED: Grant: European Society of Pediatric Research; UNRELATED: Employment: University Medical Centre Utrecht; Payment for Lectures Including Service on Speakers Bureaus, Comments: I teach a cranial ultrasound course every year in London*; Royalties: I am a co-author of 2 books and an associate editor of another book: 1) Govaert P, de Vries L. An Atlas of Neonatal Brain Sonography, 2nd ed. (CDM 182-183), Mac Keith Press. ISBN: 978-1-898683-56-8, July 2010; 2) Hellström-Westas L, de Vries LS, Rosen I. An Atlas of Amplitude-Integrated EEGs in the Newborn. 2nd ed. Informa Health, London; August 2008; ISBN 9781841846491; 3) Inder TE, Darras BT, de Vries LS, de Plessis AJ, Neil JJ, Perlman JM. Volpe's Neurology of the Newborn. 6th ed.* Walter Knirsch-RELATED: Grant: European Society of Pediatric Research; Support for Travel to Meetings for the Study or Other Purposes, Comments: travel costs. John Simpson—Grant: European Society of Pediatric Research; UNRELATED: Consultancy: Philips Healthcare, Comments: I have undertaken consultant work for Philips related to fetal cardiac imaging, nothing about brain imaging; Payment for Lectures Including Service on Speakers Bureaus: Canon Medical Systems, Comments: I received honorariums for lecturing on fetal cardiology. Christopher J. Kelly—RELATED: Grant: European Society of Pediatric Research, British Heart Foundation.* Sophie Arulkumaran—RELATED: Grant. European Society of Pediatric Research, British Heart Foundation (FS/15/55/ 31649).* Serena J. Counsell—RELATED: Grant: European Society of Pediatric Research, Medical Research Council Action for Medical Research, British Heart Foundation*; UNRELATED: Consultancy: IXICO Ltd.* Manon Benders-RELATED: Grant: European Society of Pediatric Research, ZonMw CRUCIAL-TRIAL, Stichting Hartekind*; UNRELATED: Consultancy: Chiesi stem cells.* *Money paid to the institution.

\section{REFERENCES}

1. Hoffman JI, Kaplan S. The incidence of congenital heart disease. J Am Coll Cardiol 2002;39:1890-900 CrossRef Medline

2. Moons P, Bovijn L, Budts W, et al. Temporal trends in survival to adulthood among patients born with congenital heart disease from 1970 to 1992 in Belgium. Circulation 2010;122:2264-72 CrossRef Medline

3. GBD 2017 Congenital Heart Disease Collaborators. Global, regional, and national burden of congenital heart disease, 1990-2017: a systematic analysis for the Global Burden of Disease Study 2017. Lancet Child Adolesc Heal 2020;4:185-200

4. Liamlahi R, Latal B. Neurodevelopmental outcome of children with congenital heart disease. Handb Clin Neurol 2019;162:329-45 CrossRef Medline

5. Miller SP, McQuillen PS, Hamrick S, et al. Abnormal brain development in newborns with congenital heart disease. $N$ Engl J Med 2007 Nov;357:1928-38 CrossRef

6. Newburger JW, Bellinger DC. Brain injury in congenital heart disease. Circulation. 2006;113:183-85 CrossRef

7. Johnston MV. Congenital heart disease and brain injury. $N$ Engl J Med 2007;357:1971-73 CrossRef Medline 
8. Galli KK, Zimmerman RA, Jarvik GP, et al. Periventricular leukomalacia is common after neonatal cardiac surgery. J Thorac Cardiovasc Surg 2004;127:692-704 CrossRef Medline

9. Beca J, Gunn J, Coleman L, et al. Pre-operative brain injury in newborn infants with transposition of the great arteries occurs at rates similar to other complex congenital heart disease and is not related to balloon atrial septostomy. J Am Coll Cardiol 2009;53:1807-11 CrossRef Medline

10. Chen J, Zimmerman RA, Jarvik GP, et al. Perioperative stroke in infants undergoing open heart operations for congenital heart disease. Ann Thorac Surg 2009;88:823-29 CrossRef Medline

11. Bertholdt S, Latal B, Liamlahi R, et al; Research Group Heart and Brain. Cerebral lesions on magnetic resonance imaging correlate with preoperative neurological status in neonates undergoing cardiopulmonary bypass surgery. Eur J Cardiothorac Surg 2014;45:62532 CrossRef Medline

12. Liu AY, Zimmerman RA, Haselgrove JC, et al. Diffusion-weighted imaging in the evaluation of watershed hypoxic-ischemic brain injury in pediatric patients. Neuroradiology 2001;43:918-26 CrossRef Medline

13. Mahle WT, Tavani F, Zimmerman RA, et al. An MRI study of neurological injury before and after congenital heart surgery. Circulation 2002;106:I109-14 Medline

14. Soul JS, Robertson RL, Wypij D, et al. Subtle hemorrhagic brain injury is associated with neurodevelopmental impairment in infants with repaired congenital heart disease. J Thorac Cardiovasc Surg 2009;138:374-81 CrossRef Medline

15. Claessens NHP, Algra SO, Jansen NJ, et al. Clinical and neuroimaging characteristics of cerebral sinovenous thrombosis in neonates undergoing cardiac surgery. J Thorac Cardiovasc Surg 2018;155:115058 CrossRef Medline

16. Licht DJ, Shera DM, Clancy RR, et al. Brain maturation is delayed in infants with complex congenital heart defects. J Thorac Cardiovasc Surg 2009;137:529-36; discussion 536-37 CrossRef Medline

17. McQuillen PS, Barkovich AJ, Hamrick SE, et al. Temporal and anatomic risk profile of brain injury with neonatal repair of congenital heart defects. Stroke 2007;38:736-41 CrossRef Medline

18. Petit CJ, Rome JJ, Wernovsky G, et al. Preoperative brain injury in transposition of the great arteries is associated with oxygenation and time to surgery, not balloon atrial septostomy. Circulation 2009;119:709-16 CrossRef Medline

19. Beca J, Gunn JK, Coleman L, et al. New white matter brain injury after infant heart surgery is associated with diagnostic group and the use of circulatory arrest. Circulation 2013;127:971-79 CrossRef Medline

20. Dimitropoulos A, McQuillen PS, Sethi V, et al. Brain injury and development in newborns with critical congenital heart disease. Neurology 2013;81:241-48 CrossRef Medline

21. Algra SO, Haas F, Poskitt KJ, et al. Minimizing the risk of preoperative brain injury in neonates with aortic arch obstruction. J Pediatr 2014;165:1116-22 CrossRef Medline

22. Claessens NH, Algra SO, Ouwehand TL, et al; CHD Lifespan Study Group Utrecht. Perioperative neonatal brain injury is associated with worse school-age neurodevelopment in children with critical congenital heart disease. Dev Med Child Neurol 2018;60:1052-58 CrossRef Medline

23. Peyvandi S, Chau V, Guo T, et al. Neonatal brain injury and timing of neurodevelopmental assessment in patients with congenital heart disease. J Am Coll Cardiol 2018;71:1986-96 CrossRef Medline

24. Kelly CJ, Arulkumaran S, Pereira CT, et al. Neuroimaging findings in newborns with congenital heart disease prior to surgery: an observational study. Arch Dis Child 2019;104:1042-48 CrossRef Medline

25. Lim JM, Porayette P, Marini D, et al. Associations between age at arterial switch operation, brain growth, and development in infants with transposition of the great arteries. Circulation 2019;139:272838 CrossRef Medline

26. Claessens $\mathrm{NH}$, Chau V, de Vries LS, et al. Brain injury in infants with critical congenital heart disease: insights from two clinical cohorts with different practice approaches. J Pediatr 2019;215:7582.e2 CrossRef Medline

27. Claessens NH, Khalili N, Isgum I, et al. Brain and CSF volumes in fetuses and neonates with antenatal diagnosis of critical congenital heart disease: a longitudinal MRI Study. AJNR Am J Neuroradiol 2019;40:885-91 CrossRef Medline

28. Yushkevich PA, Piven J, Hazlett HC, et al. User-guided 3D active contour segmentation of anatomical structures: significantly improved efficiency and reliability. Neuroimage 2006;31:1116-28 CrossRef Medline

29. Fedorov A, Beichel R, Kalpathy-Cramer J, et al. 3D slicer as an image computing platform for the quantitative imaging network. Magn Reson Imaging 2012;30:1323-41 CrossRef Medline

30. Moeskops P, Viergever MA, Mendrik AM, et al. Automatic segmentation of MR brain images with a convolutional neural network. IEEE Trans Med Imaging 2016;35:1252-61 CrossRef Medline

31. Makropoulos A, Robinson EC, Schuh A, et al. The developing human connectome project: a minimal processing pipeline for neonatal cortical surface reconstruction. Neuroimage 2018;173:88112 CrossRef Medline

32. Wagenaar $N$, Martinez-Biarge $M$, van der $A a N E$, et al. Neurodevelopment after perinatal arterial ischemic stroke. Pediatrics 2018;142:e20174164 CrossRef Medline

33. Papile LA, Burstein J, Burstein R, et al. Incidence and evolution of subependymal and intraventricular hemorrhage: a study of infants with birth weights less than 1,500 gm. J Pediatr 1978;92:529-34 CrossRef Medline

34. Guo T, Chau V, Peyvandi S, et al. White matter injury in term neonates with congenital heart diseases: topology and comparison with preterm newborns. Neuroimage 2019;185:742-49 CrossRef Medline

35. Algra SO, Jansen NJ, van der Tweel I, et al. Neurological injury after neonatal cardiac surgery: a randomized, controlled trial of 2 perfusion techniques. Circulation 2014;129:224-33 CrossRef Medline

36. Block AJ, McQuillen PS, Chau V, et al. Clinically silent preoperative brain injuries do not worsen with surgery in neonates with congenital heart disease. J Thorac Cardiovasc Surg 2010;140:550-57 CrossRef Medline

37. Kelly P, Hayman R, Shekerdemian LS, et al. Subdural hemorrhage and hypoxia in infants with congenital heart disease. Pediatrics 2014;134:e773v81 CrossRef Medline

38. Looney CB, Smith JK, Merck LH, et al. Intracranial hemorrhage in asymptomatic neonates: prevalence on MR images and relationship to obstetric and neonatal risk factors. Radiology 2007;242:53541 CrossRef Medline

39. Whitby EH, Griffiths PD, Rutter S, et al. Frequency and natural history of subdural haemorrhages in babies and relation to obstetric factors. Lancet 2004;363:846-51 CrossRef Medline

40. Tavani F, Zimmerman RA, Clancy RR, et al. Incidental intracranial hemorrhage after uncomplicated birth: MRI before and after neonatal heart surgery. Neuroradiology 2003;45:253-58 CrossRef Medline

41. Hunt R, Hoffman CM, Emani S, et al. Elevated preoperative von Willebrand factor is associated with perioperative thrombosis in infants and neonates with congenital heart disease. J Thromb Haemost 2017;15:2306-16 CrossRef Medline

42. Kinney HC, Panigrahy A, Newburger JW, et al. Hypoxic-ischemic brain injury in infants with congenital heart disease dying after cardiac surgery. Acta Neuropathol 2005;110:563-78 CrossRef Medline 